\title{
Mutter oder Vater haben Krebs - wie geht es den Kindern?
}

Wim Nieuwenboom

Korrespondenz:

Wim Nieuwenboom

Fachhochschule

Nordwestschweiz

Hochschule für Soziale Arbeit

Stahlrain 2

CH-5200 Brugg

Tel. 0564628823

wim.nieuwenboom@fhnw.ch

\section{Einleitung}

Eine Krebserkrankung stellt für die betroffene Person ein kritisches Lebensereignis dar. Die Diagnose wie auch die Krise, die damit ausgelöst werden kann, bleiben nicht ohne Auswirkungen auf das soziale Umfeld. Nicht nur die Patienten selbst sind betroffen, auch ihre Familien werden davon berührt. Die Krankheit, die sie begleitenden Ängste und Belastungen seitens der betroffenen Eltern können dazu führen, dass die Kinder in eine schwierige Lage geraten. Dies lassen die wenigen Forschungsergebnisse, die hierzu vorliegen, deutlich werden. Auch jüngere Kinder spüren bereits die Belastungen, die von der Krankheit ausgehen. Weil die Eltern aber oft selber schon zu sehr von der Krankheit in Anspruch genommen sind oder nicht selten auch ihre eigenen Kinder schonen wollen, indem sie nicht über die Krankheit sprechen oder sie sogar $\mathrm{zu}$ verheimlichen versuchen, bleiben den Kindern oft wenig Möglichkeiten, Fragen über die elterliche Krankheit zu stellen, ihre Gefühle auszudrücken und die Situation aktiv zu verarbeiten. Damit kann die Krankheit der Eltern zu einem Anlass für gleichzeitig oder später auftretende psychosoziale Anpassungsprobleme und soziale Auffälligkeit werden. Die beobachteten Probleme variieren von (Trennungs-)Angst und depressivem Rückzug bei jüngeren Kindern, bis zum Ausagieren und zu Schulleistungsabfall bei älteren Kindern. Bei der Entstehung von psychosozialen Problemen und/oder depressiver Symptomatik scheint es eine Wechselwirkung zwischen dem Geschlecht des Elternteils und dem Geschlecht und dem Alter des Kindes zu geben: Insbesondere bilden die pubertierenden Mädchen krebskranker Mütter eine Risikogruppe (vgl. dazu [1, 2]). In der Pubertät können zudem Ablösungsschwierigkeiten entstehen, weil zu Hause eine erhöhte Verantwortungsübernahme gefordert wird.

Leider wurde die Problematik der Kinder seitens der Wissenschaft, zumindest im deutschsprachigen Raum, bislang nur spärlich untersucht [3]. Insbesondere über psychosoziale Probleme von Kindern von neudiagnostizierten Patientinnen und Patienten ist noch wenig bekannt. Einerseits ist zu erwarten, dass die Proble- matik in dieser Situation noch wenig ausgeprägt ist. Andererseits würde eine Früherkennung einer allfälligen Problematik die Möglichkeit für ein rechtzeitiges Eingreifen eröffnen. Angesichts des wachsenden Interesses am Thema der Angehörigenproblematik beabsichtigt nun eine aktuelle Untersuchung, einen Beitrag zur Schliessung dieser Wissenslücke zu leisten. Das Projekt wird von Forschenden der Psychiatrischen Dienste Aargau, der Hochschule für Soziale Arbeit der Fachhochschule Nordwestschweiz und des Kantonsspitals Aarau durchgeführt.

\section{Ziel, Fragestellung und Untersuchungsgruppe}

Ziel der Untersuchung ist es, vertiefte Erkenntnisse zu psychosozialen Problemen von Kindern mit einem krebskranken Elternteil zu gewinnen. Die Ergebnisse sollen die Aufmerksamkeit für die Folgen erhöhen, die eine Krebserkrankung von Eltern für ihre Kinder haben kann, und damit einen Beitrag dazu leisten, diesem Aspekt in der Betreuung der Patientinnen und Patienten, die Kinder haben, vermehrt Beachtung schenken zu können.

Die Studie untersucht, ob bei Kindern kurz nach der Krebsdiagnose eines Elternteils und im ersten Jahr danach psychosoziale Probleme auftreten und allenfalls, welcher Art die Probleme sind. Sie fragt zudem nach Risiko- und Schutzfaktoren, insbesondere innerhalb des Familiensystems, sowie auch nach den Bewältigungsstrategien der Kinder.

Untersucht werden Kinder, die zum Zeitpunkt der Krebsdiagnose eines Elternteils im Alter zwischen 6 und 12 Jahren sind. Ein Einschlusskriterium (nebst einem «informed consent» und genügenden Deutschkenntnissen) ist, dass die Diagnose beim Elternteil nicht mehr als 8 Wochen zurückliegt.

\section{Design der Studie}

Die Untersuchung ist als eine prospektive Längsschnittstudie mit Erhebungen zu mehreren Messzeitpunkten sowohl bei der Gruppe der Kinder mit einem krebskranken Elternteil als auch bei der Vergleichsgruppe konzipiert. Die Be- 
fragung der Kinder und der Angehörigen ihrer Kernfamilie bzw. ihres familienähnlichen Systems wird mittels standardisierten Face-to-face-Interviews durchgeführt. Dabei wird angestrebt, mindestens 50 Kinder mit einen krebskranken Elternteil in die Studie einzubeziehen. Die quantitativen Daten werden in Verfahren der beschreibenden und schliessenden Statistik ausgewertet.

Bei den Kindern mit krebskrankem Elternteil findet die erste Befragung innerhalb der ersten zwei Monate nach der Diagnose statt, die zweite Befragung 6 Monate und die dritte Befragung 12 Monate nach der Erstbefragung. Die Kinder und ihre Angehörigen in der Vergleichsgruppe werden zu zwei Messzeitpunkten befragt, die 12 Monate auseinanderliegen.

Die Rekrutierung der Kinder erfolgt über die Ansprache der Eltern. Zur Rekrutierung der Kinder mit einem krebskranken Elternteil werden Krebspatientinnen und -patienten mit Kindern im Alter von 6 bis 12 Jahren von der behandelnden Ärztin bzw. dem behandelnden Arzt zur Teilnahme an der Studie eingeladen. In einem kurzen Gespräch wird ihnen das Ziel und das Vorgehen erläutert. Nachdem die Patientinnen und Patienten und ihre Partner/innen das Einverständnis ihrer Kinder eingeholt und schriftlich ihre Zustimmung gegeben haben, wird ein Termin vereinbart, wobei darauf geachtet wird, dass die Kinder über die Krankheit des Elternteils zuvor informiert worden sind. Zur Rekrutierung der Kinder der Kontrollgruppe wurden Eltern von Schülern in repräsentativ ausgewählten Schulklassen über die Untersuchung informiert und angefragt, an der Untersuchung als Teil der Vergleichsgruppe teilzunehmen. Bei jenen Eltern, die ihre Zustimmung schriftlich abgegeben hatten, folgte eine telefonische Terminvereinbarung.

Die Befragung aller Kinder findet zu Hause statt, also in deren vertrauten Umgebung. Die Befragung dauert 45 bis 60 Minuten. Dabei kommen Erhebungsinstrumente zum Einsatz, die für Kinder dieser Altersgruppe konzipiert wurden und sich in der Untersuchung von Kindern bewährt haben:

- FAST (Familiensystemtest nach Gehring: Familiendarstellung mit Hilfe von Holzfiguren mit einem anschliessenden Interview);

- «Dominique» (ein Bilderbuchtest der sich an der DSM-III/IV orientiert);

- DIKJ (deutsche Version des CDI, Depressionsinventar für Kinder nach Beck).

Die Eltern machen in einer halbstündigen Befragung ebenfalls eine Familiendarstellung mit dem
FAST. Bei den Eltern werden zusätzlich folgende Instrumente eingesetzt:

- SDQ (Strengths and Difficulties Questionnaire nach Goodman: Beurteilungsskala für Verhaltensauffälligkeiten des Kindes in den letzten 6 Monaten). Dieses Verfahren wurde den gebräuchlicheren CBCL (Child Behavior Checklist) vorgezogen, da dieser bei deutlich weniger Aufwand die gleichen Informationen erfasst und in den Items vermehrt positive Verhaltensweisen (Ressourcen) mit einbezieht.

- SCL-9 nach Klaghofer (Kurzversion des SCL90: Symptomcheckliste nach Derogatis: Erfassung der psychischen Belastung der Eltern).

Bei den Befragungen zu den späteren Messzeitpunkten werden dieselben Erhebungsinstrumente eingesetzt. Die relevanten soziodemographischen Daten und ein einfaches Genogramm werden nur in der ersten Befragung erhoben.

Parallel zu den Befragungen wird jeweils zu jedem Befragungszeitpunkt von der behandelnden Ärztin bzw. dem behandelnden Arzt ein Kurzfragebogen zum Gesundheitszustand der Patientin bzw. des Patienten ausgefüllt und weitergeleitet.

\section{Ethik}

Die Studie wurde der Ethikkommission des Kantons Aargau vorgelegt und von dieser ohne Vorbehalte genehmigt.

\section{Aktueller Stand}

Die Erfahrung zeigt, dass die Studie bei den Familien auf gute Akzeptanz stösst. Dies zeigt sich auch daran, dass sich von den 12 bisher in die Studie aufgenommenen Familien keine aus der Untersuchung zurückgezogen hat. Die bisherigen Interviews fanden in einer guten Atmosphäre statt, wobei das anfängliche Zögern, das bei den Kindern vor der Befragungssituation in einigen Fällen zu beobachten war, rasch einer grossen Offenheit zum Erzählen gewichen ist. Diese Beobachtung gilt auch für die Vergleichsgruppe. Das Studiendesign erweist sich damit als machbar und für die Befragten als zumutbar.

Die Rekrutierung von Kindern für die Vergleichsgruppe ist abgeschlossen. Die Rekrutierung der Kinder mit einem krebskranken Elternteil ist vor einiger Zeit angelaufen, aber noch nicht abgeschlossen. Sie basierte bislang auf zwei Kliniken und erweist sich als anspruchsvoll. Die Erfahrungen zeigen, dass viele Patientinnen und Patienten den Eingangskriterien nicht genügten, da sie oft später als 8 Wochen nach der Erstdiagnose oder gar erst im Fall von Rezidiven in diese 
Kliniken eintreten. Aus diesem Grund werden weitere Kliniken einbezogen und die Rekrutierung über niedergelassene Ärztinnen und Ärzte wie auch Beratungsstellen und Selbsthilfegruppen angestrebt.

Zurzeit sucht die Studiengruppe die Zusammenarbeit mit weiteren niedergelassenen Ärzten und Ärztinnen sowie Beratungsstellen, die bereit sind, an der Rekrutierung mitzuwirken.

\section{Projektinformation}

Die Untersuchung wird von der Krebsliga Schweiz, der Aargauischen Krebsliga und der Stiftung für Forschung im Gesundheitswesen des Kantons Aargau finanziell unterstützt. Die Studie wurde von Dr. med. Jürg Unger-Köppel (Facharzt für Kinder- und Jugendpsychiatrie, CEO, Psychiatrische Dienste Aargau) initiiert und wird von Evelyne Jaquenoud Sirot MSc (Leiterin Forschung, Psychiatrische Dienste Aargau) und Prof. Dr. phil. Daniel Gredig (Hochschule für Soziale Arbeit, FH Nordwestschweiz) verantwortet. Beteiligt am Projekt sind weiter Dr. med. Martin Wernli und lic. phil. Alfred Künzler der Abteilung Onkologie und Hämatologie des Kan- tonsspitals Aarau. Über die letztgenannte Stelle sowie über die Frauenabteilung und über die onkologische Abteilung des Universitätsspitals Zürich werden zurzeit Kinder bzw. ihre Familien angefragt.

Kontaktperson für die Studie ist Wim Nieuwenboom (Hochschule für Soziale Arbeit, FH Nordwestschweiz), der auch für die Durchführung der Untersuchung zuständig ist. Interessierte mit weiterführenden Fragen sowie Ärztinnen und Ärzte, die bei der Rekrutierung mitzuwirken bereit sind, sind eingeladen, sich mit ihm in Verbindung zu setzen.

\section{Literatur}

1 Compas BE, Worsham NL, Epping-Jordan JE, Grant KE, Mireault G, Howell DC, Malcarne VL. When Mom or Dad has cancer: markers of psychological distress in cancer patients, spouses, and children. Health Psychology 1994;13(6):507-15.

2 Lichtman RR, Taylor SE, Wood JV, Bluming AZ, Dosik GM, Leibowitz RL. Relations with children after breast cancer: the mother-daughter-relationship at risk. J Psychosoc Oncol 1984;2(3/4):1-19.

3 Ärztliche Praxis Spezial 14/2002. www. aerztlichepraxis.de. 